\title{
Micronutrients and cancer aetiology: the epidemiological evidence
}

\author{
BY TIMOTHY KEY \\ Imperial Cancer Research Fund, Cancer Epidemiology Unit, Gibson Building, \\ Radcliffe Infirmary, Oxford $O X 26 \mathrm{HE}$
}

This presentation is a discussion of the possible role of micronutrients in the aetiology of common human cancers. It is not a systematic review of the topic. Useful published reviews include a comprehensive review of fruit and vegetables in relation to cancer (Block et al. 1992), a careful review of cancer risk and serum micronutrients in prospective studies (Comstock et al. 1992), and the recent compilation prepared for the Nutrition Society (UK Nutritional Epidemiology Group for the Nutrition Society, 1993).

\section{MICRONUTRIENTS IN HUMAN DIETS}

With the possible exception of Fe (see p. 609), the underlying hypothesis is that people consuming larger amounts of certain micronutrients may have a lower risk of developing certain types of cancer. It should be borne in mind, therefore, that the classic micronutrient deficiency diseases occur almost entirely in poor people, mostly in Third World countries. Therefore, if one ignores other possible complicating factors, it might be expected that any cancers due to a low intake of micronutrients would be more common in poor countries than in rich countries. This is true for some cancers for which the role of micronutrients is under investigation (for example, cancers of the oesophagus, stomach and cervix) but is the reverse of the situation for other cancers (colo-rectal, breast, prostate).

\section{INTERPRETATION OF EPIDEMIOLOGICAL STUDIES OF MICRONUTRIENTS}

The conclusions drawn in the present discussion rely on epidemiological studies rather than laboratory studies. The advantage of epidemiological studies is that they can measure the association between an exposure such as micronutrient intake and the development of cancer. One of their disadvantages is that they may be unable to distinguish between dietary components which are strongly correlated with each other. For example, individuals with diets high in fruit and vegetables typically have high intakes of several micronutrients including carotene, ascorbic acid and pteroylglutamate (folate) (for example, see Freudenheim et al. 1991), and measuring these chemicals in blood samples does not necessarily help in distinguishing between them. Apparent differences in the strength of the association of risk with different nutrients must be interpreted with great caution because the strongest associations may be due simply to more accurate measurement of some nutrients. For many micronutrients, especially those which occur together in fruit and vegetables, epidemiological studies may never be able to identify the true risk factor. Intervention trials can test the effects of specific micronutrients, but few have yet been completed and they are limited to testing a small number of nutrients over a few years of follow-up, much shorter than the likely duration of development of most cancers. 


\section{THE COMMONEST CANCERS WORLDWIDE}

Pisani et al. (1993) have estimated the number of deaths due to cancer worldwide in 1985. The seven commonest sites in men were lung, stomach, liver, colon-rectum, oesophagus, mouth-pharynx and prostate, and those in women were breast, stomach, cervix, colon-rectum, lung, ovary and oesophagus. The present review discusses each of these sites in turn, with the exception of cancers of the liver and ovary (for which there is little information on micronutrients) and oral cancer (for which the epidemiology is somewhat similar to that for oesophageal cancer).

\section{Lung cancer}

Cigarette smoking is the major cause of lung cancer and most of the epidemiology of lung cancer can be explained by smoking patterns, but a large number of observational epidemiological studies have shown, almost without exception, that fruit and vegetable consumption is inversely related to lung cancer risk (Block et al. 1992; van Poppel, 1993). Smokers eat less fruit and vegetables than non-smokers (Gregory et al. 1990), but the inverse association of risk with fruit and vegetables remains after adjustment for smoking (Dorgan et al. 1993) and has been observed among non-smokers (Mayne et al. 1994). Several studies have reported that risk is inversely related to estimated consumption or serum concentrations of carotenoids (for example, Knekt et al. 1991; Stähelin et al. 1991), but as noted previously this would be expected because these nutrients are supplied by fruit and vegetables. The results of the observational studies could be due to any of the constituents of fruit and vegetables.

The results recently published by The Alpha-Tocopherol, Beta-Carotene Cancer Prevention Study Group (1994) provided no evidence for a protective effect of either $\alpha$-tocopherol or $\beta$-carotene after 5 to 8 years supplementation. Indeed. the incidence of lung cancer was higher in men who received $\beta$-carotene than in those who did not. Although it is possible that a protective effect of carotene could have been missed because the follow-up was too short, this trial has certainly weakened the hypothesis that $\beta$-carotene reduces lung cancer risk and it now appears somewhat more likely that the protective effect of fruit and vegetables is due to carotenoids other than $\beta$-carotene, to ascorbic acid, or to other nutrient or non-nutrient constituents of these foods. Le Marchand et al. (1993) analysed risk in relation to intake of five different carotenoids and reported that risk was inversely related to intake of $\alpha$-carotene, $\beta$-carotene and lutein but was not related to intake of lycopene or $\beta$-cryptoxanthin. They also reported that a high intake of vegetables was more strongly protective than a high intake of the three apparently protective carotenoids, suggesting that components of vegetables other than carotenoids, or carotenoids not assessed, might be protective.

It is possible that some of the apparent protective effect of fruit and vegetables might be due to an association of these foods with other dietary characteristics such as a low intake of saturated fat and cholesterol, since these factors have been observed to be positively associated with lung cancer risk (Shekelle et al. 1991; Alavanja et al. 1993). Whether micronutrients affect risk or not, there is no doubt that cigarette smoking is the major preventable cause of lung cancer. 


\section{Oesophageal cancer}

The incidence of oesophageal cancer is moderate in most Western populations but high in parts of Asia and Africa and extremely high in certain restricted localities; the incidence rates in Kazakhstan and northern Iran are the highest observed in general populations for any type of cancer anywhere in the world (Cook-Mozaffari, 1989). The range of incidence rates in men between different regions of the world is about 500-fold, which is much larger than the range for other common cancers (Cook-Mozaffari, 1989).

The major identified causes of oesophageal cancer in Western countries are smoking and alcohol consumption, and Day \& Munoz (1982) have estimated that these two factors probably account for about $90 \%$ of cases in Europe, North America and the Caribbean and explain most of the variation with time, place and population group within these countries. However, the extremely high rates of oesophageal cancer which occur in both men and women in areas such as Iran are not due to smoking or alcohol intake. The high-risk populations typically have diets composed of a small range of foods, mostly plant foods, and may have low intakes of several micronutrients such as retinol, $\beta$-carotene, riboflavin, ascorbic acid and vitamin E (Blot et al. 1993). Around the south of the Caspian Sea, for example, little besides bread, tea and sugar is eaten for most of the year and consequently the diet is very low in animal protein, retinol, riboflavin and ascorbic acid, and clinical signs of riboflavin deficiency are widespread (Cook-Mozaffari, 1989). In a comparison of low- and high-risk areas within China, Thurnham et al. (1985) reported that riboflavin status (but not vitamin A or Zn status) was significantly worse in the high-risk area. Other dietary factors characteristic of some high-incidence populations may also increase risk; for example irritation of the oesophageal mucosa by very hot food and drinks and by dry scratchy bread and maizemeal (Cook-Mozaffari, 1989).

Almost all the analytical epidemiological studies have found that a high consumption of fresh fruit and vegetables and, therefore, of carotenoids and ascorbic acid, is associated with a reduced risk of oesophageal cancer (Steinmetz \& Potter, 1991; Block et al. 1992). A recent case-control study in Hong Kong, a high-risk area, found a tenfold reduction in risk for frequent consumption of citrus fruits (Cheng et al. 1992). There was also a significant increase in risk for a high intake of pickled vegetables, which are rich in $N$-nitroso compounds. The attributable risks in this study were $48 \%$ for alcohol, $44 \%$ for tobacco, $29 \%$ for pickled vegetables, $26 \%$ for a low intake of citrus fruits, $15 \%$ for a low intake of green leafy vegetables, and $14 \%$ for consumption of hot drinks and soups. These results illustrate the importance of both causative and protective dietary factors in the aetiology of this cancer.

A randomized trial has been conducted in Linxian, China, to test the effects on cancer incidence and mortality of four micronutrient preparations: retinol and $\mathrm{Zn}$; riboflavin and niacin; ascorbic acid and Mo; $\beta$-carotene, Se and $\alpha$-tocopherol (Blot et al. 1993). The relative risks for death from oesophageal cancer when these four preparations were given were: $0.93,0.90,1.05$ and 0.96 respectively, all not significant. Thus, there was no strong evidence that any of the micronutrients tested can prevent oesophageal cancer, but the follow-up period was only 5 years. It should also be noted that the validation study conducted within this trial showed that the nutritional status for riboflavin and for ascorbic acid improved substantially during the trial among the subjects who did not receive these supplements, thus weakening the contrast between groups. In a parallel trial of a multiple vitamin and mineral supplement among people with oesophageal 
dysplasia the relative risk of death from oesophageal cancer among those receiving the supplement was $0 \cdot 84$; this reduction was not statistically significant but the intervention period was only 6 years ( $\mathrm{Li}$ et al. 1993).

There was no evidence for a protective effect of either $\beta$-carotene or vitamin $E$ in the Finnish study (Alpha-Tocopherol, Beta-Carotene Cancer Prevention Study Group, 1994).

It is very likely that a substantial proportion of the high incidence of oesophageal cancer in some populations is due to dietary deficiency of micronutrients such as vitamin A, riboflavin, ascorbic acid and $\mathrm{Zn}$, but the identity of these factors is not certain. The attributable risk for micronutrient deficiencies is also not well-established, and some of the high risk in some populations is due to other factors such as dietary $N$-nitroso compounds.

\section{Stomach cancer}

Until it was recently overtaken by lung cancer, stomach cancer was the most common cancer in the world. It is still a major cause of death in most countries, but rates are generally much higher in poor countries than in rich countries and both incidence and mortality rates have fallen dramatically over the past 40 years in virtually all countries for which statistics are available (Muñoz, 1988).

Observational epidemiological studies have shown convincing evidence of a protective effect of fruit and vegetables (Forman, 1991; Block et al. 1992) and of high plasma levels of carotene and ascorbic acid (Stähelin et al. 1991). Correa's (1992) model proposes that ascorbic acid may inhibit the intermediate stages of carcinogenesis by reducing intragastric nitrosation, and that $\beta$-carotene might inhibit the later stages of carcinogenesis, perhaps by scavenging free radicals and opening intercellular gap junction communications (Correa, 1992). The recent trial among the general population in Linxian, China, reported a significant reduction in risk (relative risk 0.79 ) after 5 years of supplementation with a combination of $\beta$-carotene, vitamin $\mathrm{E}$ and $\mathrm{Se}$, but no protective effect was observed for ascorbic acid given with Mo (relative risk 1.09; Blot et al. 1993). As noted previously, this trial is weak for the ascorbic acid group because ascorbic acid intake rose substantially in the control group. There was no evidence for a protective effect of $\beta$-carotene (or $\alpha$-tocopherol) after 5 to 8 years supplementation in the AlphaTocopherol, Beta-Carotene Cancer Prevention Study (Alpha-Tocopherol, BetaCarotene Cancer Prevention Study Group, 1994).

Although the evidence is incomplete, the hypothesis that ascorbic acid reduces the risk for stomach cancer is strong. It is possible that some of the temporal trends and international differences in rates of stomach cancer could be due to differences in nutrient intake, especially for ascorbic acid, but there is no doubt that other factors increase risk, including dietary salt, some preserved foods, and infection with the bacterium Helicobacter pylori (Forman, 1991).

\section{Colo-rectal cancer}

Of all the common cancers, the certainty that dietary factors are aetiologically important is probably greatest for colo-rectal cancer. Doll \& Peto (1981) suggested that dietary factors could account for $90 \%$ of the variation in rates between countries. However, 
there are several plausible dietary hypotheses and it has not yet been possible to identify the most important factors. There is considerable theoretical and epidemiological evidence in support of the following broad dietary hypotheses: that risk is increased by a high intake of meat or animal fat and that risk is decreased by a high intake of dietary fibre or fruit and vegetables (Potter, 1992).

Observational studies of fruit and vegetables and the risk of colo-rectal cancer have provided considerable evidence of a protective effect (Block et al. 1992). As would be expected, there are also several reports of a low risk associated with a high intake or plasma level of carotenoids (van Poppel, 1993).

Even for micronutrients supplied by fruit and vegetables the picture is particularly complex for colo-rectal cancer, since there is considerable interest in the possibility that folate, as well as the antioxidant nutrients, may have a protective effect. This is supported by the role of folate as an essential cofactor in the production of s-adenosylmethionine, the primary methyl donor in the body, by the observed accumulation of DNA methylation abnormalities during the progression of human colo-rectal cancer, and by an inverse association of folate intake with the risk of adenomas (Giovannucci et al. 1993) and colo-rectal cancer (Benito et al. 1991; Freudenheim et al. 1991).

The difficulty with large-bowel cancer is not only the usual uncertainty about which of the several micronutrients in fruit and vegetables might be protective, but the likelihood that the protective factor in these foods could be non-starch polysaccharides or starch rather than any of the micronutrients. For example, in their combined analysis of thirteen case-control studies, Howe et al. (1992) found that $\beta$-carotene, ascorbic acid and fibre were each individually significantly inversely related to risk, but that after adjustment for each other only the relationship with fibre remained strong and statistically significant. It is also possible that some of the apparent protective effect of various plant foods may be due to an inverse association of their intake with that of meat and animal fat.

There is some evidence that high Fe stores may increase the risk for colo-rectal cancer. Knekt et al. (1994) reported a relative risk of 3.0 for subjects with a transferrin saturation level exceeding $60 \%$ in comparison with subjects having lower transferrin saturation levels. It has been suggested that an excess of unabsorbed Fe might promote colo-rectal cancer, but it is also possible that the observed increase in risk could be due to an association of high $\mathrm{Fe}$ stores with a high meat intake.

The nature of the effects of diet on colo-rectal cancer risk is probably the most important question in the area of diet and cancer, particularly in Western populations. The competing hypotheses, although they are somewhat interrelated, could result in substantially different messages for public health. More data are needed from large prospective studies with both accurate measurement of diet and a wide range of dietary intakes, but the almost uniformly low rates of colo-rectal cancer in poor populations suggest that a diet low in meat and animal fat and high in plant foods may be more important than individual micronutrients in determining risk.

\section{Breast cancer}

In general, breast-cancer rates are much higher in rich countries than in poor countries. Some of this difference is due to hormonal factors: women in poor countries typically 
have later menarche, earlier first birth, higher parity and lower body weight than women in rich countries, and all these factors are known to reduce breast-cancer risk by shortening the duration of exposure of the breasts to ovarian hormones, reducing the usual levels of these hormones, and (in the case of parity) by altering the structure of the breasts (Pike et al. 1983; Key \& Pike, 1988). However, the inability of these traditional risk factors to explain all the geographic and temporal variation in breast-cancer rates, and the strong international correlation between breast-cancer rates and some dietary factors, has led to numerous studies of diet and breast-cancer risk. The evidence that any micronutrients have an important effect on breast-cancer risk is weak. The best data are probably for Se level, which was reported to show an inverse geographic correlation with breast-cancer rates and which can reduce mammary tumour incidence in experimental animals when administered at close to toxic doses. However, results from five cohort studies which measured Se in blood or nails and which were conducted in countries with varying Se levels (Britain, Finland, The Netherlands and USA) have produced almost uniformly negative results, with the exception of a possible (but not statistically significant) protective association in Finland (Hunter \& Willett, 1993).

Epidemiological evidence relating to the other antioxidant nutrients is surprisingly sparse (in comparison with the number of reports on fat) and generally inconsistent. There is some evidence that retinol, carotenoids or ascorbic acid may be inversely associated with breast-cancer risk (Hunter \& Willett, 1993), but the size of the observed effects has typically been very small and their statistical significance largely due to the large size of the studies. For example, Hunter et al. (1993) reported a highly significant $(P=0.001)$ inverse trend between total vitamin A intake and breast-cancer risk, but women with the highest intake had only $16 \%$ lower risk than those with the lowest intake. Measurement error in nutritional epidemiology can cause serious underestimation of the size of risks, but differences in risk as small as this must be suspected of being due to confounding by some other factor.

Research on breast cancer has provided an interesting example of a situation in which antioxidants might have an adverse effect. In a study of the growth of human breast-cancer tissue in athymic nude mice, Gonzalez et al. (1993) reported that increasing the intake of fish oil inhibited cancer growth, but that this inhibition was largely reversed by the addition of the antioxidants $\alpha$-tocopherol and tertiary butylhydroquinone. They suggested that the suppression of cancer growth by fish oil was due to accumulation of lipid peroxidation products within the tumour and that this was reduced by the antioxidants. These results should not be overinterpreted, but they do show that antioxidants might have unexpected effects.

The role of diet in the aetiology of breast cancer remains to be established. As well as fat and energy intake, current areas of interest include the possible protective antioestrogenic effects of non-starch polysaccharides and phyto-oestrogens. Although further research is needed, it does not appear to be very likely that micronutrients are an important determinant of risk.

\section{Cervical cancer}

Seven of the eight studies reviewed by Block et al. (1992) reported an inverse association between fruit and vegetable intake and the risk for cervical cancer. There has been considerable interest in folate and there is some evidence that folate deficiency may 
increase the risk for cervical dysplasia in association with human papillomavirus-16 infection (Butterworth et al. 1992), but studies of invasive cervical cancer have produced more support for a role of carotene or of ascorbic acid than folate. For example, in the large case-control study of Herrero et al. (1991), high intakes of $\beta$-carotene, other carotenoids and vitamin $C$ were associated with odds ratios of $0 \cdot 6-0 \cdot 7$, but the odds ratio for high intake of folate-rich foods was 1.0 .

There is little doubt that the major aetiological factor for cervical cancer is infection with human papillomavirus (Schiffman, 1992). It is possible that low intakes of carotene, ascorbic acid or other factors in fruit and vegetables may also increase risk, but careful consideration of the possibility of confounding by other factors such as hygiene is needed before this conclusion is drawn.

\section{Prostate cancer}

Prostate cancer is rare in east Asia, moderately common in southern and western Europe, and very common in northern Europe and in north America, especially among the black population. Little is known about the causes of prostate cancer, but there is quite consistent evidence that an increased risk is associated with diets high in meat or animal fat (Le Marchand et al. 1994).

Several studies have examined the relationship between carotene or carotene-rich foods and risk. Some authors have reported their results as suggesting that carotene is protective, and some as suggesting that carotene increases risk. Furthermore, recent studies have reported results separately for older and younger men, and shown that carotene increases (or decreases) risk in one age-group but not in the other. However, systematic review of these studies shows that there is no evidence that risk is related to carotene intake overall and no evidence for a systematic variation of risk in relation to age at diagnosis (Key, 1994).

The evidence does not support a role for carotene in the aetiology of prostate cancer, but there is some recent evidence suggesting that two other micronutrients might be important: Corder et al. (1993) reported significantly lower serum concentrations of 1,25-dihydroxycholecalciferol (but not 25-hydroxycholecalciferol) in men who subsequently developed prostate cancer than in control men; the Alpha-Tocopherol, Beta-Carotene Cancer Prevention Study Group (1994) reported a significantly lower mortality from prostate cancer in men who were given $50 \mathrm{mg} \alpha$-tocopherol daily for 5 years than in men who were not given $\alpha$-tocopherol. These two reports raise the possibility of protective effects for these micronutrients, but they require confirmation.

\section{COMMENT ON SMOKING, CANCER AND ANTIOXIDANTS}

It has been shown repeatedly in a number of different populations that, in comparison with non-smokers, cigarette smokers have a lower average consumption of fruits and vegetables and, therefore, of carotene and ascorbic acid. Furthermore, the metabolic effects of smoking appear to result in lower plasma concentrations of these nutrients in smokers than in non-smokers, even at the same level of intake (Gregory et al. 1990), and there is some evidence that smokers have increased free-radical-mediated lipid peroxidation (Duthie et al. 1993). Cigarette smoking has been shown to cause cancers of the lung, lip, mouth, oesophagus, pancreas, renal pelvis and bladder, and is associated with 
(but not definitely causal for) cancers of the stomach, liver and cervix (International Agency for Research on Cancer, 1987). Many of the studies of diet and cancer discussed in the present review have been interpreted by researchers as supporting the hypothesis that antioxidant micronutrients may reduce the risk of cancer at some sites. However, of the cancers discussed in the present article, smoking is not an established cause of colo-rectal cancer, breast cancer or prostate cancer. This is an additional piece of evidence which suggests that the antioxidants affected by smoking ( $\beta$-carotene, ascorbic acid and perhaps others) are not important protective factors for cancer at these sites.

\section{SUMMARY}

Micronutrient deficiencies occur most commonly in poor countries and, therefore, are most likely to be associated with cancers common in these countries. Epidemiological studies are hampered by inaccurate measurement of micronutrient intake and by the correlations between intakes of many nutrients. The strongest evidence for a protective effect of micronutrients is for oesophageal cancer. The identity of the micronutrients is not certain, but may include retinol, riboflavin, ascorbic acid and $\mathrm{Zn}$; alcohol, smoking and dietary nitrosamines increase the risk for oesophageal cancer. For stomach cancer there is good evidence that fruit and vegetables are protective. The protective effect of these foods might be largely due to ascorbic acid, but other nutrients and non-nutrients may also be important; the risk for stomach cancer is increased by salt, some types of preserved foods, and by infection of the stomach with the bacterium Helicobacter pylori. The risk for lung cancer appears to be reduced by a high intake of fruit and vegetables, but it is not clear which agents are responsible and the major cause of lung cancer is cigarette smoking. Diet is probably the major determinant of the risk for colo-rectal cancer; there is evidence that fruit and vegetables and fibre reduce risk and that meat and animal fat increase risk, but there is no convincing evidence that these relationships are mediated by micronutrients. The risk for cervical cancer is inversely related to fruit and vegetable consumption and, therefore, to consumption of carotenoids and ascorbic acid, but the major cause of this cancer is human papillomavirus and it is not yet clear whether the dietary associations indicate a true protective effect or whether they are due to confounding by other variables. The evidence that micronutrients are important in the aetiology of either breast cancer or prostate cancer is weak, but the possible roles of 1,25 -dihydroxycholecalciferol and $\alpha$-tocopherol in prostate cancer require further study.

\section{REFERENCES}

Alavanja, M. C. R., Brown, C. C., Swanson, C. \& Brownson, R. C. (1993). Saturated fat intake and lung cancer risk among nonsmoking women in Missouri. Journal of the National Cancer Institute 85, 1906-1915.

Benito, E., Stigglebout, A., Bosch, F. X., Obrador, A., Kaldor, J., Mulet, M. \& Muñoz, N. (1991). Nutritional factors in colorectal cancer risk: a case-control study in Majorca. International Journal of Cancer 49, 161-167.

Block, G., Patterson, B. \& Subar, A. (1992). Fruit, vegetables and cancer prevention: a review of the epidemiological evidence. Nutrition and Cancer 18, 1-29.

Blot. W. J., Li, J.-Y., Taylor, P. R., Guo, W., Dawsey, S., Wang. G.-Q., Yang, C. S., Zheng, S.-F., Gail, M., I.i, G.-Y., Yu, Y., Liu, B.-Q., Tangrea, J., Sun, Y.-H., Liu. F., Fraumeni, J. F., Zhang, Y.-H. \& Li, B. (1993). Nutrition intervention trials in Linxian, China: supplementation with specific vitamin/mineral combinations, cancer incidence, and disease specific mortality in the general population. Journal of the National Cancer Institute 85, 1483-1492. 
Butterworth, C. E. Jr, Hatch, K. D., Macaluso, M., Cole, P., Sauberlich, H. E., Soong, S.-J., Borst, M. \& Baker, V. V. (1992). Folate deficiency and cervical dysplasia. Journal of the American Medical Association 267, 528-533.

Cheng, K. K., Day, N. E., Duffy, S. W., Lam, T. H., Fok, M. \& Wong, J. (1992). Pickled vegetables in the aetiology of oesophageal cancer in Hong Kong Chinese. Lancet 339, 1314-1318.

Comstock, G. W., Bush, T. L. \& Helzlsouer, K. (1992). Serum retinol, beta-carotene, vitamin E, and selenium as related to subsequent cancer of specific sites. American Journal of Epiderniology 135, 115-121.

Cook-Mozaffari, P. (1989). Epidemiology and predisposing factors. In Management of Oesophageal Carcinoma, pp. 31-56 [R. L. Hurt, editor]. London: Springer-Verlag.

Corder, E. H., Guess, H. A., Hulka, B. S., Friedman, G. D., Sadler, M., Vollmer, R. T., Lobaugh, B., Drezner, M. K., Vogelman, J. H. \& Orentreich, N. (1993). Vitamin D and prostate cancer: a prediagnostic study with stored sera. Cancer Epidemiology, Biomarkers and Prevention 2, 467-472.

Correa, P. (1992). Human gastric carcinogenesis: a multistep and multifactorial process. First American Cancer Society award lecture on cancer epidemiology and prevention. Cancer Research 52, 6735-6740.

Day, N. E. \& Muñoz, N. (1982). Cancer of the oesophagus. In Cancer Epidemiology and Prevention [D. Schottenfeld and J. F. Fraumeni, editors]. New York: Saunders.

Doll, R. \& Peto, R. (1981). The causes of cancer: quantitative estimates of avoidable risks of cancer in the United States today. Journal of the National Cancer Institute 66, 1191-1308.

Dorgan, J. F., Ziegler, R. G., Schoenberg, J. B., Hartge, P., McAdams, M. J., Falk, R. T., Wilcox, H. B. \& Shaw, G. L. (1993). Race and sex differences in associations of vegetables, fruits and carotenoids with lung cancer risk in New Jersey (United States). Cancer Causes and Control 4, 273-281.

Duthie, G. G., Arthur, J. R., Beattie, J. A. G., Brown, K. M., Morrice, P. C., Robertson, J. D., Shortt, C. T., Walker, K. A. \& James, W. P. T. (1993). Cigarette smoking, antioxidants, lipid peroxidation and coronary heart disease. Annals of the New York Academy of Sciences 686, 120-129.

Forman, D. (1991). The etiology of gastric cancer. In Relevance to Human Cancer of N-Nitroso Compounds, Tobacco Smoke and Mycotoxins, pp. 22-32 [1. K. O'Neill, J. Chen and H. Bartsch, editors]. Lyon: International Agency for Research on Cancer.

Freudenheim, J. L., Graham, S., Marshall, J. R., Haughey, B. P., Cholewinski, S. \& Wilkinson, G. (1991). Folate intake and carcinogenesis of the colon and rectum. International Journal of Epidemiology 20, 368-374.

Giovannucci, E., Stampfer, M. J., Colditz, G. A., Rimm, E. B., Trichopoulos, D., Rosner, B. A., Speizer, F. E. \& Willett, W. C. (1993). Folate, methionine, and alcohol intake and risk of colorectal adenoma. Journal of the National Cancer Institute 85, 875-884.

Gonzalez, M. J., Schemmel, R. A., Dugan, L., Gray, J. I. \& Welsch, C. W. (1993). Dietary fish oil inhibits human breast carcinoma growth: a function of increased lipid peroxidation. Lipids 28, 827-832.

Gregory, J., Foster, K., Tyler, H. \& Wiseman, M. (1990). The Dietary and Nutritional Survey of British Adults. London: H.M. Stationery Office.

Herrero, R., Potischman, N., Brinton, L. A., Reeves, W. C., Brenes, M. M., Tenorio, F., de Britton, R. C. \& Gaitan, E. (1991). A case-control study in nutrient status and invasive cervical cancer. American Journal of Epidemiology 134, 1335-1346.

Howe, G. R., Benito, E., Castelleto, R., Cornée, J., Estève, J., Gallagher, R. P., Iscovich, J. M., Deng-ao, J., Kaaks, R., Kune, G. A., Kune, S., L'Abbé, K. A., Lee, H. P., Lee, M., Miller, A. B., Peters, R. K., Potter, J. D., Riboli, E., Slattery, M. L., Trichopoulos, D., Tuyns, A., Tzonou, A., Whittemore, A. S., Wu-Williams, A. H. \& Shu, Z. (1992). Dietary intake of fiber and decreased risk of cancers of the colon and rectum: evidence from the combined analysis of 13 case-control studies. Journal of the National Cancer Institute 84, 1887-1896.

Hunter, D. J., Manson, J. E., Colditz, G. A., Stampfer, M. J., Rosner, B., Hennekens, C. H., Speizer, F. E. \& Willett, W. C. (1993). A prospective study of the intake of vitamins C, E, and A and the risk of breast cancer. New England Journal of Medicine 329, 234-240.

Hunter, D. J. \& Willett, W. C. (1993). Diet, body size, and breast cancer. Epidemiologic Reviews 15, $110-132$.

International Agency for Research on Cancer (1987). Monographs on the Evaluation of Carcinogenic Risk to Humans: An Updating of IARC Monographs Volumes 1 to 42. Lyon: IARC.

Key, T. J. A. (1994). Risk factors for prostate cancer. Cancer Surveys (In the Press).

Key, T. J. A. \& Pike, M. C. (1988). The role of oestrogens and progestagens in the epidemiology and prevention of breast cancer. European Journal of Cancer and Clinical Oncology 24, 29-43.

Knekt, P., Järvinen, R., Seppänen, R., Rissanen, A., Aromaa, A., Heinonen, O. P., Albanes, D., Heinonen, M., Pukkala, E. \& Teppo, L. (1991). Dietary antioxidants and the risk of lung cancer. American Journal of Epidemiology 134, 471-479. 
Knekt, P., Järvinen, R., Seppänen, R., Rissanen, A., Aromaa, A., Heinonen, O. P., Albanes, D., Heinonen, M., Pukkala, E. \& Teppo, L. (1991). Dietary antioxidants and the risk of lung cancer. American Journal of Epidemiology 134, 471-479.

Knekt, P., Reunanen, A., Takkunen, H., Aromaa, A., Heliövaara, M. \& Hakulinen, T. (1994). Body iron stores and risk of cancer. International Journal of Cancer 56, 379-382.

Le Marchand, L., Hankin, J. H., Kolonel, L. N., Beecher, G. R.. Wilkens, L. R. \& Zhao, L. P. (1993). Intake of specific carotenoids and lung cancer risk. Cancer Epidemiology, Biomarkers and Prevention 2, $183-187$.

Le Marchand, L., Kolonel, L. N., Wilkens, L. R., Myers, B. C. \& Hirohata, T. (1994). Animal fat consumption and prostate cancer: a prospective study in Hawaii. Epidemiology 5, 276-282.

Li, J.-Y., Taylor, P. R., Li, B., Dawsey, S., Wang, G.-Q., Ershow, A. G., Guo, W., Liu, S.-F., Yang, C. S., Shen, Q., Wang, W., Mark, S. D., Zou, X.-N., Greenwald, P., Wun, Y.-P. \& Blot, W. J. (1993). Nutrition intervention trials in Linxian, China: multiple vitamin/mineral supplementation, cancer incidence and disease-specific mortality among adults with esophageal dysplasia. Journal of the National Cancer Institute 85, 1492-1498.

Mayne, S. T., Janerich, D. T., Greenwald, P., Chorost, S., Tucci, C., Zaman, M. B., Melamed, M. R., Kiely, M. \& McKneally, M. F. (1994). Dietary beta carotene and lung cancer risk in U.S. nonsmokers. Journal of the National Cancer Institute 86, 33-38.

Muñoz, N. (1988). Descriptive epidemiology of gastric cancer. In Gastric Carcinogenesis, pp. 51-69 [P. I. Reed and M. J. Hill, editors]. Amsterdam: Excerpta Medica.

Pike, M. C., Krailo, M. D., Henderson, B. E., Casagrande, J. T. \& Hoel, D. G. (1983). 'Hormonal' risk factors, 'breast tissue age' and the age-incidence of breast cancer. Nature 303, 767-770.

Pisani, P., Parkin, D. M. \& Ferlay, J. (1993). Estimates of the worldwide mortality from eighteen major cancers in 1985. Implications for prevention and projections of future burden. International Journal of Cancer 55, 891-903.

Potter, J. D. (1992). Reconciling the epidemiology, physiology and molecular biology of colon cancer. Journal of the American Medical Association 268, 1573-1577.

Schiffman, M. H. (1992). Recent progress in defining the epidemiology of human papillomavirus infection and cervical neoplasia. Journal of the National Cancer Institute 84, 394-398.

Shekelle, R. B.. Rossof, A. H. \& Stamler, J. (1991). Dietary cholesterol and incidence of lung cancer: the Western Electric study. American Journal of Epidemiology 134, 480-484.

Stähelin, H. B., Gey, K. F., Eichholzer, M., Ludin. E., Bernasconi, F., Thurneysen, J. \& Brubacher, G. (1991). Plasma antioxidant vitamins and subsequent cancer mortality in the 12-year follow-up of the prospective Basel study. American Journal of Epidemiology 133, 766-775.

Steinmetz, K. A. \& Potter, J. D. (1991). Vegetables, fruit and cancer. I. Epidemiology (review). Cancer Causes and Control 5, 325-357.

The Alpha-Tocopherol, Beta-Carotene Cancer Prevention Study Group (1994). The effect of vitamin E and beta carotene on the incidence of lung cancer and other cancers in male smokers. New England Journal of Medicine 330, 1029-1035.

Thurnham, D. I., Zheng, S., Muñoz, N., Crespi, M., Grassi, A., Hambidge, K. M. \& Chai, T.-F. (1985). Comparison of riboflavin, vitamin $A$ and zinc status of Chinese populations at high and low risk for esophageal cancer. Nutrition and Cancer 7, 131-143.

UK Nutritional Epidemiology Group for the Nutrition Society (1993). Diet and Cancer: a Review of the Epidemiological Literature. London: The Nutrition Society.

van Poppel, G. (1993). Carotenoids and cancer: an update with emphasis on human intervention studies. European Journal of Cancer 29A. 1335-1344. 